Review

\title{
Advantages and Limitations of Current Microgravity Platforms for Space Biology Research
}

\author{
Francesca Ferranti, Marta Del Bianco * and Claudia Pacelli
}

check for

updates

Citation: Ferranti, F.; Del Bianco, M.; Pacelli, C. Advantages and Limitations of Current Microgravity Platforms for Space Biology Research. Appl. Sci. 2021, 11, 68. https://dx.doi.org/ 10.3390/app11010068

Received: 30 October 2020

Accepted: 23 November 2020

Published: 23 December 2020

Publisher's Note: MDPI stays neutral with regard to jurisdictional claims in published maps and institutional affiliations.

Copyright: (c) 2020 by the authors. Licensee MDPI, Basel, Switzerland. This article is an open access article distributed under the terms and conditions of the Creative Commons Attribution (CC BY) license (https: / / creativecommons.org/ licenses/by/4.0/).
Italian Space Agency, Via del Politecnico snc, 00133 Rome, Italy; francesca.ferranti@asi.it (F.F.); claudia.pacelli@asi.it (C.P.)

* Correspondence: marta.delbianco@asi.it
Keywords: microgravity; ground-based facility; international Space station; clinostat; RPM; bed rest; CubeSat

\section{Introduction}

Space exploration has deep scientific implications that are not solely astrophysical, but also biological. Space biology is concerned with discovering the origin of life on Earth and finding extra-terrestrial forms of life, but also understanding how the conditions on Earth shape life and how organisms are affected by the Space environment (microgravity, radiation, etc.). Fruit flies were the first organisms sent to Space in 1947. Many experiments followed before the first human Space flight by Yuri Gagarin in 1961. Since 2000, technological progress has allowed humans to constantly inhabit Space on board of the International Space Station (ISS). As missions have progressed, so has the knowledge of how living organisms respond to the conditions of Space.

The Space environment is not compatible with life as known on Earth; a near vacuum, with temperatures ranging from hundreds of degrees to near absolute zero, and a conspicuous dose of solar and cosmic radiations. Bacteria, tardigrades, fungi, and other forms of life have survived in dormant form to outer Space exposure [1-6], but life needs protection and insulation from these conditions to thrive away from Earth. The variations in the gravitational force have also been shown to have profound effects on living organisms. Life on Earth has evolved under the influence of constant gravity; it provides a directional stimulus once thought to be perceived only by gravitropic organisms, like plants [7]. It is now known that gravity influences life at physiological and cellular level, affecting development, membrane exchange, cell metabolism, and growth. Alterations of the gravitational force are perceived as stress. In humans, for example, microgravity induces important physiological alterations, such as muscle atrophy [8], bone loss [9], 
cardiovascular deconditioning [10,11], immunological [12,13], cerebrovascular [14] and cognitive alterations [15,16], and metabolic problems [17]. In some cases, these physiological alterations can adversely affect performance and health in astronauts, both during and after the mission. Indeed, these adaptive responses seem to be partially reversible after return to Earth, but can take up to years, if ever, to fully recover $[8,18]$. Moreover, future missions beyond Low Earth Orbit (LEO) will engage astronauts for longer and these effects could become even more critical.

The importance of an adequate understanding of the physiological responses of living organisms to microgravity has grown since the beginning of human Space exploration. Scientists have developed ground-based microgravity simulators to study the physiological and molecular responses to altered gravity, and to develop effective countermeasures for spaceflight. Depending on the scientific question, different experimental platforms are available and experimental applications can range in size from molecules to humans. There are different ways to reproduce the effects of microgravity on Earth. Research in this field was given a head start by the pioneers of plant gravitropism, starting from T.A. Knight at the beginning of the XIX century. The so-called microgravity simulators do not reproduce microgravity conditions, but rather achieve omnilateral stimulation by randomizing the direction of gravity over time (clinostat and random positioning machine) or compensate the gravitational force with a counteracting force (magnetic levitation). Microgravity analogues just reproduce the effects of microgravity on physiological responses. Analogues are bed rest and bed rest associated with head-down tilt (HDT), water immersion (neutral buoyancy), and total or partial body suspensions. Even though simulated microgravity conditions only reproduced some effects observed in a true microgravity environment, they represent effective, affordable, and readily available test beds. Microgravity can be transiently achieved with several non-orbiting facilities based on free-fall, which include drop towers, parabolic flights, and small rockets, which are widely used for short-term experiments. Long-term microgravity experiments can be performed on orbiting facilities, despite the constraints and limitations of spaceflight missions. The first modular Space station was the Russian Mir, which was deorbited in 2001, and, for the past two decades, scientists have been able to use the ISS. Smaller orbiting facilities have been available since 1957 (i.e., biosatellites) and, more recently, CubeSats have been used to test hypotheses in constant, real microgravity. Each of these platforms is preferably used in specific biological systems. This is determined by experimental design factors, like level and quality of microgravity, duration, and sample size limitations, that must be taken into consideration when designing experiments and interpreting results.

The aim of this review is to give a general overview of the currently available facilities for the study of microgravity. An attempt was made to critically discuss the advantages and limitations of each class of facilities, giving recommendations for appropriate selection of samples and experimental design. In particular, we highlighted the practical aspects of biological payload integration on the ISS.

\section{Ground Microgravity Simulators}

\subsection{Clinostat $1 D / 2 D / R W V$}

The simplest and most affordable tool used to manipulate gravity on Earth is a clinostat. Clinostats were introduced in the late 19th century by the pioneers of plant gravitropism [19] and, through the years, have evolved to be applied to new technologies and new needs. Clinostats simulate the absence of a directional gravity input by averaging the gravity vector through rotation over time [20] (Table 1). 
Table 1. Specifications of ground microgravity simulators. RWV, rotating wall vessel; RPM, random positioning machine.

\begin{tabular}{lccc}
\hline & 1D/2D Clinostat/RWV & 3D Clinostat/RPM & Diamagnetic Levitation \\
\hline Microgravity Duration & Hours to weeks & Hours to weeks & Minutes to hours \\
Microgravity Quality & $\leq 10^{-3} \mathrm{~g}$ & $10^{-4} \mathrm{~g}$ & $<10^{-2} \mathrm{~g}$ \\
Hypogravity & $\mathrm{Y}$ & $\mathrm{Y}$ & $\mathrm{Y}$ \\
Biological System & Cells, microbes, plants & Cells, microbes, plants & Cells, microbes, \\
Cost & Low & Low & plants, animals \\
Accessibility & Easy & Easy & Medium \\
\hline
\end{tabular}

Depending on the rotation speed and the number of rotation axes, basic clinostats fall into different classes and find application in different fields. Clinostats rotate around one axis (1D or $2 \mathrm{D}$ clinostat) or two axes (3D clinostats). In 1D clinostats, the vertical axis of the organism is a continuation of the rotation axis of the clinostat (Figure 1a), while in 2D clinostats, the specimen is rotated on a plane perpendicular to the rotation axis (Figure 1b). Slow revolutions $(0.3-3 \mathrm{rpm})$ are common for whole plants to minimise the chances of the centrifugal acceleration mimicking gravity [20]. For the same reason, fast revolving clinostats can only be used for cell suspensions in very narrow vessels (few $\mathrm{mm}$ ). In these clinostats, the fast rotation (30-150 rpm) eliminates the effect of gravity by stabilizing the fluid around the cell. Rotating wall vessels (RWVs), instead, are larger than the fast-rotating clinostats (5-20 cm diameter) and rotate at 10-20 rpm around the horizontal axis. In RWV, the rotation frequency is matched to the sedimentation velocity, so that the cells remain centred in the vessel and rotate around their central axis [21].

a

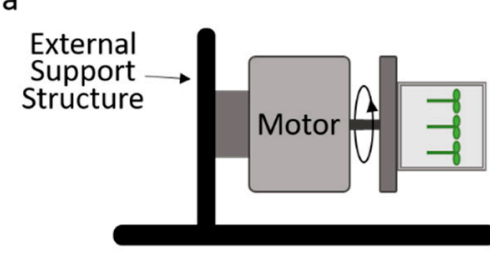

C

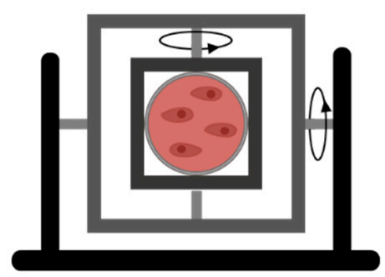

b

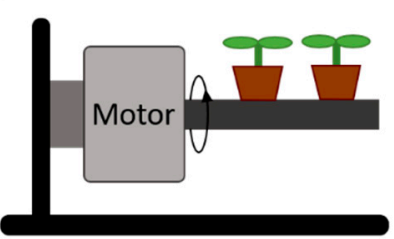

d

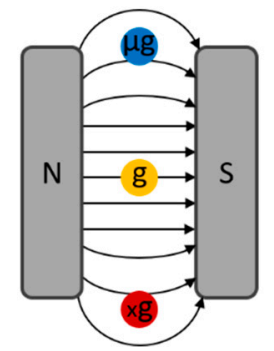

Figure 1. Ground microgravity simulators. Schematic representation of (a) 1D clinostat; (b) 2D clinostat; (c) 3D clinostat/RPM (random positioning machine); and (d) diamagnetic levitation system. Clinostats and RPMs simulate the absence of a directional gravity input by averaging the gravity vector through rotation around one or two axes over time. Diamagnetic levitation compensates gravity at molecular level, by balancing the weight of water and biological tissues with the repulsive force exerted by a magnetic field. Within the magnetic field, a specimen can experience from zero $(\mu \mathrm{g})$ to twice the gravity force $(\mathrm{xg})$ depending on its position within the magnetic field gradient.

The simplicity of a clinostat setup allows for great adaptability to different experimental applications and clinostats are also used to mimic hypogravity. This can be achieved simply by non-uniform rotation, by tilting the rotation axis so that a fraction of the gravity vector is not averaged, or by pairing clinostats with centrifuges [22-26]. This has been used, for example, to demonstrate that the plant gravitropic response is guided by the inclination 
and not the force of the gravity stimulus [25]. Moreover, cameras and microscopes have been implemented on clinostats to allow for live imaging during clinorotation [25].

Clinostats cancel the directionality of the gravity vector, but are unable to reproduce actual microgravity. Indeed, gravity may be averaged, but is never eliminated and the shift in weight distribution can cause mechanical and bending stress. For these reasons, the results obtained on a clinostat must be viewed in the right prospective. Moreover, in plants, the distinction between gravitropism and gravisensitivity must be considered when choosing the type of clinostat. When studying gravisensitivity, two axes rotation is to be preferred, even if 2D clinostats can also be used. For the study of gravitropism and architecture in plants, 1D clinostats should be used, as tropic responsiveness has radial symmetry around the shoot-root axis. Despite their limitations, clinostats remain an invaluable tool to study Space condition on Earth for their versatility and cost efficiency.

\section{2. $R P M$}

Three-dimensional clinostats are constituted by two gimbal-mounted frames that are turned independently by two dedicated motors (Figure 1c). They were first introduced in Japan by T. Hoson for plant research and later improved by Dutch Space (former Fokker Space) in the Netherlands [27]. The term 3D clinostat is appropriate only if the frames operate with constant speed and direction; if speed and direction are different and randomized, it takes the name of a "random positioning machine" (RPM) [28]. Dedicated algorithms drive frame rotation, such that the biological samples, fixed on the inner frame, are constantly reoriented and the gravity vector is averaged over time. Thus, even if $1 \mathrm{~g}$ is constantly acting on the sample, the averaged gravity vector is close to zero [29]. If the rotation is sufficiently faster than the response time of the biological system, the continuous reorientation will cause the sample to experience a state similar to microgravity [30].

Rotation induces accelerative forces. Therefore, sample positioning within the RPM is a critical aspect to consider when using this simulator. Where the two rotational axes cross, the forces acting on the sample are negligible; further from the center, the forces acting on the outer edges of the sample become more relevant [27]. Therefore, the choice of an RPM setup imposes limits to sample volume. In addition, compared with static and real microgravity experiments, sample rotation introduces additional fluid motion in the culture flask, producing shear forces and enhancing convection [31]. To reduce shear stress, moderate rotations should be combined with smooth transitions [32]. Enhanced convection leads to improved nutrients and gas provision and waste product removal, which allow to work with higher cell densities. Higher cell density and gravitational unloading increase the chance of cell-cell interaction and formation of multicellular aggregates [33]. Thus, RPM can also be used to induce three-dimensional growth and to obtain spheroid-like structure [30,34]. Another practical aspect to consider when using the RPM is to avoid the formation of air bubbles in the liquid medium. Air bubbles produce unwanted fluid motion and shear stress [27,35] and can cause the detachment of adherent cells [30].

Even if this platform has been used extensively to simulate microgravity for Space biology studies (preparatory tests for flight-experiments, pre- and post-flight experiments, etc.), the RPM has been shown to mimic microgravity effects only partially. Indeed, discrepancies in gene regulation were found between cells exposed to the RPM and those exposed to real microgravity in Space [30]. Therefore, data from RPM experiments must be interpreted carefully and, when possible, compared to experiments in real microgravity.

In summary, in order to obtain reliable data with the RPM, the experimental conditions must be carefully set. However, depending on the combination of rotation speed and the distance from the center, high quality microgravity conditions can be obtained, in the order of $10^{-4} \mathrm{~g}$ (Table 1) [34]. Moreover, thanks to recently introduced algorithms, RPM machines can average Earth gravity partially to simulate Moon- $(0.16 \mathrm{~g})$ or Mars-like $(0.33 \mathrm{~g})$ gravity conditions (Table 1) [30]. 


\subsection{Diamagnetic Levitation}

Another approach to simulate microgravity on Earth is the use of diamagnetic levitation. Diamagnetic levitation compensates gravity at a molecular level, by balancing the weight of water and biological tissues with the repulsive force exerted by a magnetic field (Figure 1d). While electromagnets are bigger and more powerful, they tend to heat to temperatures incompatible with standard room-temperature growth conditions, thus requiring supercooling and limiting the duration of the experiment to few hours. Permanent magnets are relatively easier to use, but their size greatly limits the type of sample that can be used in the experiment. Nonetheless, diamagnetic levitation has not only been used to study microgravity in cell culture, but also in whole organisms like plants, frogs, and fruit flies (Table 1) [36], among others.

Diamagnetic levitation can also be used to simulate partial gravity. Indeed, the magnetic field generated by magnets is a gradient (Figure 1d). Within this gradient, a specimen can experience from zero to twice the gravity force. By placing the sample at different points in this gradient, intermediate values can be achieved, including the gravitational force of the Moon and Mars (Table 1) [37].

Diamagnetic levitation is very effective at compensating gravity. However, it should be noted that samples withstand a strong magnetic field, in addition to microgravity, and this may influence the organism. Differences in the diamagnetic properties of cellular and tissue components and the relative position in the magnetic gradient of the sample parts will result in discrepancies in the applied force. Humans have reported vertigo-like sensations and apparent perception of movement when in the proximity of the magnetic resonance imaging scanner strong magnetic field [38]. On a cellular level, strong magnetic fields affect the orientation of biomolecules, like DNA, tubulin, and actin [39], and subcellular components, like plant statoliths [40]. Strong magnetic fields have also been shown to change the membrane potential and influence ion-channel activity [41]. Some effects of the strong magnetic field can be distinguished from the ones due to levitation by carefully designing the controls to be performed in the parts of the gradient where the sample experiences $1 \mathrm{~g}$ acceleration (Figure 1d).

\section{Ground Microgravity Analogues}

\subsection{Bed Rest}

Bed rest is used to simulate microgravity-associated inactivity in humans, in a normal gravitational environment. Horizontal beds were initially used, but later, $6^{\circ}$ head-down tilt (HDT) bed rest was introduced by Russian scientists. HDT better simulates the head-ward fluids shifts in Space, where blood is redistributed from the legs to the head (Figure 2a) and, at present, HDT bed rest is the most common analogue for the simulation of microgravity. Long-duration bed rest normally uses volunteers and is employed to simulate the effects of microgravity on various physiological systems, mainly for bone, muscles, and cardiovasculature, as well as to test countermeasures for microgravity environment.

The duration of bed rest experiments may vary from minutes to months (up to 12 months), depending on the objectives of the study (Table 2) [42]. Healthy individuals are monitored $24 \mathrm{~h}$ per day and subjected to standardized conditions, like diet, stress, physical activity, fluid intakes, light/dark circadian cycles, etc. Bed rest is generally used for studying cardiovascular deconditioning, exercise capacity impairment, and musculoskeletal disorders [43,44]. Bed rest is also widely used to test countermeasures for musculoskeletal and cardiac atrophy, and orthostatic intolerance. Recently, focusing on long-duration missions, such as to Mars, bed rest has been used for investigating psychological behaviour and changes in the sleep-wake cycle.

Bed rest is not effective at compensating the effects of gravity on internal organs, but it remains the most effective analogue for whole body physiology research [42]. As bed rest is performed in hospitals, it is associated with medium-high costs (Table 2). However, researchers have frequent opportunities to apply for utilization of this microgravity analogue. 
Table 2. Specifications of ground microgravity analogues. ULLS, unilateral lower limb suspension; HU, hindlimb unloading.

\begin{tabular}{lccccc}
\hline & Bed Rest & ULLS & HU & Neutral Buoyancy & Dry Immersion \\
\hline Microgravity Duration & Hours to weeks & Hours to weeks & Hours to weeks & Hours & Days to months \\
Hypogravity & Yes & No & No & No & No \\
Biological System & Humans & Humans & Animals & humans & Humans \\
Cost & Medium & Low & Low & Medium & Medium \\
Accessibility & Easy & Medium & Easy & Medium & Medium \\
\hline
\end{tabular}

a

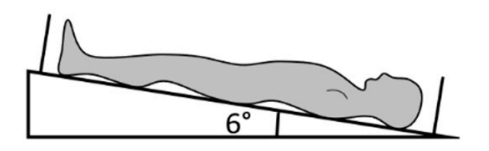

C

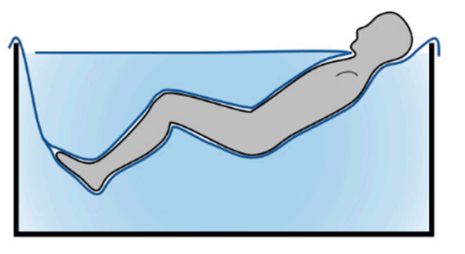

b

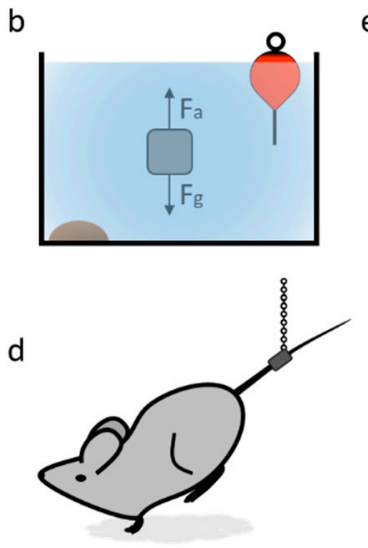

e

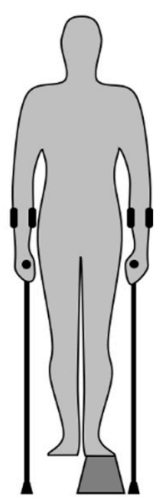

Figure 2. Ground analogues of microgravity. Schematic representation of (a) bed rest; (b) neutral buoyancy (Fg, gravitational force/weight; Fa, Archimedes/buoyancy force); (c) dry immersion; (d) rodent hindlimb unloading (HU); and (e) unilateral lower limb suspension (ULLS). Microgravity analogues reproduce the effects of microgravity on physiological responses, like muscle and bone loss, and head-ward fluids shifts.

\subsection{Neutral Buoyancy}

Neutral buoyancy occurs when a mass immersed in a medium displaces a mass of equal weight (Figure 2b). In this case, the buoyancy force will balance the gravity force. If an object has neutral buoyancy in a liquid, it will neither sink nor float, but will remain stationary. Neutral buoyancy facilities have been used since the mid-1960s at the Johnson Space Center to test procedures, to develop hardware, and for astronaut training. Astronauts use the Neutral Buoyancy Facility to practice in-flight procedures, such as extravehicular activities, and for in-flight simulations.

Neutral buoyancy has also been used in single cell organisms (Table 2). In particular, it has been used to prove the existence of statolith-independent graviperception. By matching the density of the medium to the density of the cell, scientists were able to abolish gravitydriven swimming in both Euglena and Paramecium. In these organisms, it is thought that the entire cell works as a statolith, and the mechanical stress exerted by the cell on the different faces of the cell wall is translated into positional information. It is still not clear to what extent a similar mechanism is also active in higher plants, but plants lacking sedimenting statoliths partially retain the ability to respond to gravity. Submergence has been used to study graviperception in higher plants, but the possible influence of the gas-related stress response even in semi-aquatic species makes the results difficult to interpret $[45,46]$.

\subsection{Dry Immersion}

Another widely used ground model for microgravity is dry immersion. First introduced in the early 1970s [47], dry immersion accurately and rapidly reproduces most of the physiological effects of short-term spaceflight. The test subjects lay in supine position on 
waterproof elastic fabric, immersed up to neck level into a deep bath (Figure 2c). The combination between the water buoyant force and the high elasticity of the fabric reproduces the effects of weightlessness, like a lack of vertical vascular gradient, physical inactivity, and support withdrawal.

Dry immersion reproduces the same physiological changes as HDT bed rest, but the effects can be seen after a relatively shorter period of exposure. In addition, dry immersion can be used over longer periods of time than neutral buoyancy. These characteristics have allowed scientists to perform experiments in dry immersion from a few hours up to 56 days (Table 2) [48]. Dry immersion has been used to study cardiovascular deconditioning and changes in body fluid and mineral content [49]. It is also especially important in the design of passive countermeasures, i.e., not requiring intensive motor activity of the subjects. Recently, dry immersion has also found applications for terrestrial medicine, like rehabilitation programs for premature children and in sport medicine, and for the treatment of Parkinson disease [48].

\subsection{The Unilateral Lower Limb Suspension (ULLS)}

The unilateral lower limb suspension (ULLS) method was developed and validated for studying the effects of spaceflight on muscle size and function. Suspension is achieved with the use of one tall platform shoe and crutches for ambulation, preventing the other leg from touching the ground (Figure 2e). The weight bearing withdrawal for 2-3 days affects protein metabolism of postural muscles, simulating the skeletal muscle adaptation observed in microgravity condition (Table 2) [50]. During the first 2 months ULLS, muscle mass is lost at a constant rate of about $2-3 \%$ per week. On a microscopic level, muscle loss is accompanied by a corresponding decrease in the size of individual muscle fibres. As in spaceflight, ULLS shifts the metabolism, favouring carbohydrate over fat utilization.

ULLS is a relatively inexpensive method compared with bed rest analogues and costs can be sustained by single scientific teams (Table 2). Indeed, in contrast to bed rest and neutral buoyancy, there is no need for volunteers to be confined into hospitals. As ULLS is compatible with a person's social life, it is easier to find a wider number of volunteers and increase sample size. In addition, load and overall mechanical stress are unaltered in the weight-bearing limb, which can work as a control.

There are some obvious limitations and drawbacks with ULLS. Compared with bed rest, the ULLS volunteers are not subjected to standardized conditions. Therefore, any differences in the daily routine of the volunteers can impact the response to ULLS, adding variability to the data. Moreover, compliance with the use of the system cannot be entirely controlled, even if volunteers who intentionally avoid the imposed restrictions are typically ejected early in the study. Moreover, the use of the platform shoe, especially without the crouches, can lead to dangerous falls. Serious medical issues might also stem from an increased thromboembolism risk, and predisposed subjects should thus be excluded.

\subsection{The Rodent Hindlimb Unloading ( $\mathrm{HU}$ )}

The rodent hindlimb unloading (HU) consists of the selective unloading of the hindquarters of rats or mice using tail traction, surgical pins, or body harnesses (Figure 2d) [51]. Since its development in the 1980s, HU has become a widely used and generally accepted method to simulate spaceflight-like conditions in rats and mice [52]. HU causes removal of ground reaction forces from the hindlimbs and cephalad fluid shift, similarly to what happens to astronauts in Space [5].

The validity of $\mathrm{HU}$ as an analogue was confirmed in the Cosmos 2044 mission. HU was used as a 1-g on-ground control for a 14-day long flight onboard of an unmanned biosatellite [53,54]. Despite differences in tissue collection after unloading between the two models (15-60 min for HU rats vs. 8-11 h for spaceflight rats), the results showed that $\mathrm{HU}$ and spaceflight induced similar atrophic changes in bone and muscle tissues [54,55]. Subsequently, other investigators confirmed that HU induces similar, but not always identical physiological changes to those observed in spaceflight [56]. 
Data should be interpreted cautiously when comparing the HU rodent model and spaceflight. Unlike spaceflight, in the HU model, the gravity force is still acting on the internal organs of the rodent, meaning that they exert pressure against each other, instead of free floating. Moreover, animals do not experience the stress of launch and transportation [57]. The time between animal 1-g reloading and sacrifice spans from minutes in HU to many hours for spaceflight (Table 2), which could either mitigate or mask some of the spaceflight-induced effects [51].

Environmental and physiological parameters can influence the ability of the HU to effectively induce spaceflight-like physiological responses. Animal housing, handling, sex, age (growing vs. adult), species (mouse vs. rat), and strain are important factors to consider when designing HU studies [56]. The extrapolation of conclusions from data gained in animals of different age or species should be carried out carefully. Indeed, HU could be a suitable microgravity analogue for the study of the processes responsible for growth, but not for maintenance of adult tissues. Similarly, features found in a species might not widely apply to other species (e.g., contractile properties of skeletal muscles). Another critical variable for HU studies is the length of exposure, as acute responses to short exposure and chronic adaptation after longer exposures can differ dramatically [51].

Despite its limitations, HU has several strengths compared with spaceflight. Experiments can be performed without the crew time and safety constraints arising onboard of Space vehicles. Furthermore, during the experiment, adjustments can be made without an excessive impact on cost and tissues can be sampled from sedated animals at any time. These operations, which are routine on the ground, cannot be carried out with ease in Space [56].

\section{Non-Orbiting Microgravity Facilities \\ 4.1. Drop Towers}

Drop towers provide facilities for scientific investigations that require relatively short microgravity time. In most cases, experiments are performed in an evacuated chamber to eliminate the effects of drag and friction forces. Drop towers are the most flexible and adaptable platforms for scientific applications and offer the best microgravity levels-as low as $10^{-6} \mathrm{~g}$ (Table 3, Figure 3a). The height of the tower limits the period of microgravity, which can vary from $2.2 \mathrm{~s}$ in the NASA (National Aeronautics and Space Administration) Glenn 2.2 Second Drop Tower, up to $9.5 \mathrm{~s}$ in the case of Drop Tower Catapult System in Bremen (Table 3) [58].

Drop towers are used to conduct short-term technological investigations, test instrumentation before use in Space, and preparatory experiments for long-duration missions. In biology, drop towers have been used to perform experiments focused on membrane physiology and gravitaxis. These include the study of the influence of microgravity on the electrophysiology of biological membranes and the orientation behaviour of ciliates and fish.

Common to other free-fall-based platforms, samples on drop towers undergo transient accelerations. In particular, during the loading and breaking phase, the sample can experience accelerations of up $50 \mathrm{~g}$. Payloads must thus be designed to withstand such forces.

Drop tower platforms have many advantages. They are medium-cost and have relatively easy-to-handle safety requirements. Multiple experiments can be performed within a few days and the time between experiment planning, development, and execution can be relatively short. Experiment hardware can also be changed at short notice and the setup can be adjusted or improved between drops. Drop towers also allow for realtime monitoring of experiments, as both data upload and download are available during the experiment.

Many environmental parameters can be regulated during each experimental session, like temperature and pressure, and electrical power is supplied. Centrifuges have also been paired to drop towers to reach hypogravity condition. For example, centrifuge has 
been used during drop experiments to determine the threshold values of gravisensitivity of organisms [59].

Table 3. Specifications of non-orbiting and orbiting microgravity facilities. ISS, International Space Station.

\begin{tabular}{|c|c|c|c|c|c|}
\hline & Drop Tower & Parabolic Flight & Sounding Rocket & CubeSat & ISS \\
\hline Microgravity Duration & $2.2 \mathrm{~s}$ up to $9.5 \mathrm{~s}$ & 20 s repetitive & $5-20 \mathrm{~min}$ & Weeks to months & Months to years \\
\hline Microgravity Quality & $10^{-6} \mathrm{~g}$ & $10^{-2} \mathrm{~g}$ & $\leq 10^{-4} \mathrm{~g}$ & $10^{-6} \mathrm{~g}$ & $10^{-6} \mathrm{~g}$ \\
\hline Hypogravity & Yes & Yes & No & No & Yes \\
\hline Biological System & $\begin{array}{l}\text { Cells, microbes, } \\
\text { plants }\end{array}$ & $\begin{array}{c}\text { Cells, microbes, } \\
\text { plants, animals, } \\
\text { humans }\end{array}$ & $\begin{array}{c}\text { Cells, plants, } \\
\text { microbes, animals }\end{array}$ & Microbes, plants & $\begin{array}{c}\text { Cells, microbes, } \\
\text { plants, animals, } \\
\text { humans }\end{array}$ \\
\hline Cost & Medium & Medium & Medium & Medium & High \\
\hline Accessibility & Medium & Medium & Medium & Medium & Hard \\
\hline $\begin{array}{l}\text { Late access before } \\
\text { experiment }\end{array}$ & $2 \mathrm{~h}$ & $10 \mathrm{~min}$ & $<3 \mathrm{~h}$ & $24 \mathrm{~h}$ & $24 \mathrm{~h}$ \\
\hline $\begin{array}{l}\text { Early retrieval after } \\
\text { experiment }\end{array}$ & $45 \mathrm{~min}$ & $1 \mathrm{~min}$ & $1-2 \mathrm{~h}$ & Not Applicable & $\geq 48 \mathrm{~h}$ \\
\hline
\end{tabular}
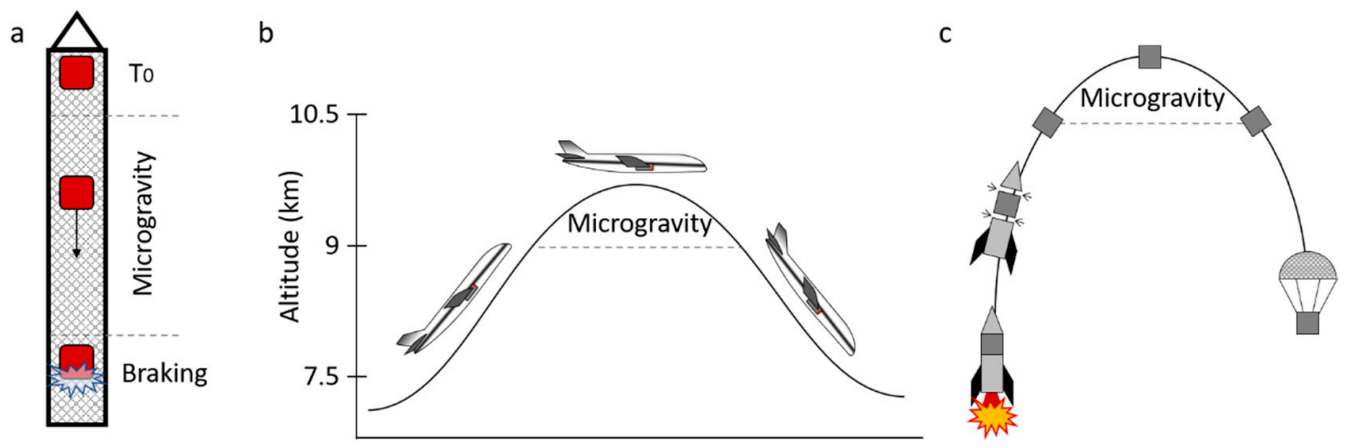

Figure 3. Non-orbiting microgravity facilities. Schematic representation of (a) drop tower; (b) parabolic flight; and (c) sounding rocket. As microgravity can only be achieved transiently, these facilities are widely used for short-term experiments.

\subsection{Parabolic Flight}

Parabolic flight allows for short-term (15-20 s maximum) exposure of humans, animals, tissues, or cells to microgravity $\left(\sim 10^{-2} \mathrm{~g}\right)$ (Table 3$)$. Such short-term simulations of microgravity are suitable for studies of acute phenomena, such as cardio-vascular and neuro-sensory response [44]. Parabolic flights are also used to test technical equipment, train astronauts for Space missions, and validate operational and experimental procedures.

A typical NASA parabolic flight lasts $3 \mathrm{~h}$. First, the plane carries experiments and crewmembers to a starting altitude of approximately $7 \mathrm{~km}$ above sea level. Then, it performs a rapid ascent at $45^{\circ}$ (pull up), traces a parabola at altitudes ranging from 7.3 to $10.4 \mathrm{~km}$ (pushover), and then descends at $45^{\circ}$ (pull out) (Figure 3b). Crew and experiments experience hypergravity (between $2 \mathrm{~g}$ and $2.5 \mathrm{~g}$ ) during the pull-up and pull-out segments. During the parabola, net acceleration drops as low as $1.5 \times 10^{-2} \mathrm{~g}$ for a brief period of time ( $>15 \mathrm{~s}$ ). ESA (European Space Agency) parabolic flights slightly differ from NASA parabolic flights in the level of hypergravity $(1.5-1.8 \mathrm{~g})$ and altitude $(7.5-8.6 \mathrm{~km})$ during pull-up and pull-out, and microgravity experienced $\left(10^{-2} \mathrm{~g}\right.$, for $\left.20 \mathrm{~s}\right)$ [60]. Experiment area available in the cabin is up to $200 \mathrm{~m}^{2}$ and electrical power is supplied during the flight. Payload can be several square meters, up to $2.9 \mathrm{~m}$ in height [59], and up to several tons in weight. A standard parabolic flight campaign consists of three flight days, each with a 3-4 h long flight with 31 parabolas in a row. After five parabolas, a 4-8 min pause allows scientists to quickly analyse the experimental run and to change parameters or experiment setup. A crew of up to 40 people is onboard, performing 12 to 15 different experiments. 
The main advantages of parabolic flights are the late access and early retrieval of the sample [61], the short time between proposal and experiment implementation (in term of months), and the medium costs (Table 3). In addition, there is the possibility of performing a series of experimental runs within three days; of using typical laboratory-type instrumentations; and of directly controlling, like for drop towers, the experiments during flight. Moreover, by changing the flight manoeuvres, it is possible to reproduce partial gravity conditions, with gravity levels corresponding to those of the Moon and Mars. Contrary, the greatest disadvantage is that microgravity is intermixed with hypergravity phases, and this must be considered when interpreting results.

\subsection{Sounding Rockets}

Sounding rockets have been used since the late 1950s for meteorological and upper atmosphere studies and, starting from 1982, they have also been used for biological experiments [62]. Sounding rockets are sub-orbital rockets that carry payloads above the atmosphere, without placing them into orbit around the Earth (Figure 3c). Sounding rockets can be used in a wide range of disciplines, like physics, materials science, and biology. In particular, scientists have used sounding rockets to study gravity-sensing mechanisms in several plants and animals.

Typically, such rockets reach an altitude of $250-350 \mathrm{~km}$, at which point the payload is released, undergoes stabilized free-fall, and finally lands by parachute (Figure 3c). A free-fall of 6-13 min and 5-20 min is obtained for the rockets used by ESA and NASA, respectively $[62,63]$. Microgravity levels are usually at or below $10^{-5} \mathrm{~g}$ (Table 3). Payloads can be quite large, thus containing several experimental modules. ESA organises sounding rockets campaigns as often as every two years.

There are several variables that must be taken into consideration when designing experiments for sounding rockets flights. Samples can experience hypergravity phases during the ascent. Moreover, the payload module outer structure can reach temperatures of $300^{\circ} \mathrm{C}$ and above, during the ascent and descent phases, respectively. An external liquid cooling loop is available for those payloads that contain temperature-sensitive experiments.

Like drop towers, sounding rockets are a good opportunity for implementing research in preparation for long-duration missions to obtain valuable scientific return at a contained cost. One of the benefits of this type of carrier is that late access to the payload is available up to less than $3 \mathrm{~h}$ before launch. This makes the use of sounding rockets compatible with studies involving samples requiring complex preparation, or focused on time-critical biological processes. Sounding rocket missions can be carried out at medium cost and payloads can be developed in as little as three months (Table 3). This rapid experimental turnover allows scientists to quickly plan new experiments, using the latest, most up-to date knowledge and technologies. Moreover, as sounding rockets are unmanned, payload safety requirements are less stringent for sounding rockets compared with parabolic flights.

\section{Orbiting Microgravity Facilities}

\subsection{CubeSats}

The CubeSat Project started in 1999 as a way of increase accessibility to Space, and now involves hundreds of groups, in both the public and private sector. A CubeSat is a modular miniaturized satellite for Space research. Each unit is a $10 \times 10 \mathrm{~cm}$ cube with a mass of no more of $1.33 \mathrm{~kg}$ and each CubeSat can be constituted of up to 12 units (Table 3, Figure 4a). The CubeSat technology is normally applied on experiments that can be miniaturized. CubeSats are employed to demonstrate spacecraft technologies intended for larger satellites and equipment for biology experiments [64,65]. In biology research, CubeSats have been employed to study the effect of the Space environment in the sporeforming bacterium Bacillus subtilis [66] and, soon, in yeast [67] and plants [64], and the stability of organic molecules. 


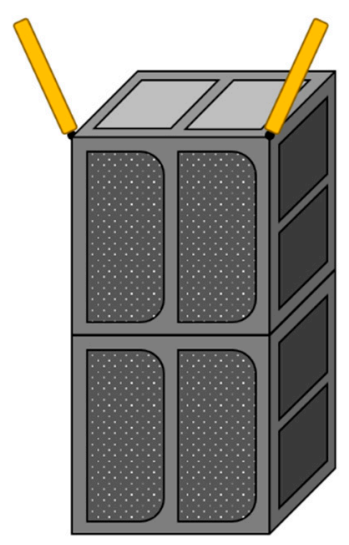

b

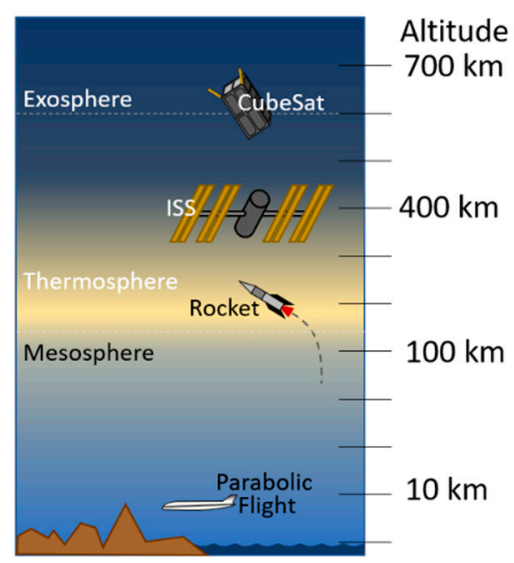

Figure 4. Schematic representation of (a) a CubeSat, a modular miniaturized satellite for Space research, composed of two units, with yellow antennas; (b) altitude in km of orbiting (CubeSats, ISS) and non-orbiting (sounding rockets, parabolic flights) facilities and atmospheric zones: the majority of CubeSats orbit at an altitude between 350 and $700 \mathrm{~km}$, but they can be at lower or higher orbits; the International Space Station (ISS) orbits at an altitude of about $400 \mathrm{~km}$; sounding sockets typically reach an altitude of 250-350 km; parabolic flights reach an altitude of $7 \mathrm{~km}$.

The technology utilised in the CubeSat is based on a standardised satellite design that uses commercial off-the-shelf (COTS) components for electronics and structure, reducing costs and development time. About 75\% of CubeSats orbit Earth at an altitude between 350 and $700 \mathrm{~km}$ [68]. They are normally powered by solar panels and can contain a control habitat for biological samples and cameras or spectrometers for image data collection [66]. CubeSats are commonly launched as secondary payloads on launch vehicles, further reducing the costs of the mission, or put in orbit by deployers on the ISS [69,70]. CubeSats, therefore, suffer from the same restrictions of other experimental opportunities on orbit, like early late access and safety standards. Moreover, if issues were to arise during orbit, they could only be managed remotely, hardware permitting, and this could be detrimental for the experiment [66]. Samples on the CubeSat are normally not retrievable.

CubeSats can also be used in missions to the Moon and Mars [71]. In May 2018, the two Mars Cube One (MarCO) provided a real-time communications link to Earth to the InSight Mars lander mission, while proving the resilience of CubeSats. Moreover, both NASA and ESA are planning to use CubeSats to support lunar exploration objectives in the next decade.

\subsection{The International Space Station}

The International Space Station (ISS) is a unique laboratory for scientific research and technology demonstration. In continuous free-fall, orbiting at $400 \mathrm{~km}$ high with a speed of $28.000 \mathrm{~km} / \mathrm{h}$, the ISS is the only platform where constant microgravity can be achieved. This has allowed scientists to investigate, among other factors, the effects of long-term exposure to microgravity on biological systems, especially humans, and test technologies needed for astronauts to live and work in such an environment. Since 2011, the International Space Exploration Coordination Group, which gathers fourteen Space agencies, has been defining the Global Exploration Roadmap (GER), a shared 20-year strategic plan for Space exploration. In the third edition of the GER, published in 2018, the International Space Station (ISS) was recognized as the steppingstone towards the expansion of human presence beyond LEO [72].

Research carried out on the ISS spans from biology to physical sciences, from Earth and Space science to educational activities and technology development. The results from ISS biological research have provided insight into the complexity of microgravity responses in whole organisms, complex cell cultures, down to protein crystals growth [73]. 
Recently, new technologies have allowed for the first onboard analysis of microbiological and genetic samples in spaceflight history. A large range of astrobiology experiments have been performed under Space conditions by pairing long-duration external exposure onboard the ISS with on-ground sample analysis after return to Earth [3,74-77]. Plant biology experiments have been conducted for the development of bioregenerative life support systems in preparation for future long-duration exploration missions, but have also highlighted some of the basic processes of plant growth on Earth. Biological and human physiological studies driven by the need to support astronaut health have yielded important results that can have a positive impact on human health on Earth. These results include new ways to mitigate bone loss; innovative wound-healing techniques; insights into bacterial behaviour; and advances in psychological stress response, disease models, and nutrition [73]. In the first twenty years of ISS activity, research on the ISS has produced almost 2500 among publications in peer-reviewed journals, conferences, and grey literature, reporting the research of more than 5000 scientists worldwide [78].

The ability to conduct research in real microgravity provides a unique chance to treat gravity, which is ubiquitous on Earth, as a variable. Nevertheless, performing experiments in Space comes with its specific challenges. In particular, the limiting factors are mass and volume, environmental requirements, time constraints, and controls. Experimental design is also constrained by operational limitations associated with launch, execution, and data collection while in Space, as well as sample return.

\subsubsection{Mass and Volume}

Access to the ISS is limited by the cost of launching and returning the mass (and associated volume) of a payload and the operational constraints. Limitations in the "up mass" and "down mass" affect the quantity of experimental material that can be launched. In particular, mass and volume restrictions impact the complexity of scientific experiments in terms of the number of variables, sample types, number of replicates, etc.

\subsubsection{Environmental Requirements}

To ensure a successful experiment and, mostly important, the safety of crew members and of the ISS environment, the on-ground preparatory risk-assessment must include all safety requirements and compliance. Research groups are normally supported by their national Space Agencies in identifying the critical points, which can vary based on the subject of the experiments. The requirements can include, for example, double containment level, non-toxic fixative for cells, fireproof material, no gas exchange, and no outgassing of hardware material. Because of the differences in gas and liquid behaviour during flight and on board the ISS, reagents easily handled in the laboratory on Earth must be strictly controlled when launched or utilized in Space.

\subsubsection{Time Constraints}

Usually, it takes about a week after delivery to the personnel conducting launch operations for a payload to be installed on the ISS. This includes pre-launch time both in the building and on the launch pad, vehicle ascent and dock with the ISS, and unloading. However, this time can vary from 4 days to longer than a week, marking a critical timeframe for many biological experiments [79]. To minimize the impact of delays, scientists are usually required to design payloads that remain viable for at least 2 weeks and to prepare a "back up" copy of the experiment in case of longer delays.

Currently, payloads do not have access to electrical power during transit, and the payload environmental control is ensured by passive stowage bags. Temperature is thus either $4{ }^{\circ} \mathrm{C}$ or ambient temperature $\left(18-29^{\circ} \mathrm{C}\right)$. A payload, therefore, needs to be designed to be able to stay dormant and viable in these environmental conditions until its installation onboard the ISS.

Once on board, most experimental procedures should be automated because crew time is not dedicated to any single experiment, but to several of them, and is thus very limited. 
For sample recovery, since the end of the Space Shuttle program, samples take a minimum time of $48 \mathrm{~h}$ from the landing to the laboratory, even in the case of the possibility to use nearby laboratories. Since the end of the shuttle program, samples take a minimum time of $48 \mathrm{~h}$ from the landing to the laboratory (in the case of the opportunity to use laboratory nearby the landing site). This is not a problem for fixed samples, but an extended exposure of living whole organisms to Earth gravity can trigger adaptive responses that can mask the previously achieved microgravity effects.

\subsubsection{Controls}

To be able to study reduced gravity as an independent experimental variable, spaceflight experiments should be accompanied by a series of control experiments performed on the same set of samples. These should include 1-g ground controls, simulated-microgravity ground controls, and in-flight 1-g controls. Ground controls are normally performed after the mission completion using the same environmental parameters registered during the whole mission, like relative humidity, temperature, and pressure. The use of an on-orbit centrifuges for in-flight 1-g controls can provide assurance that samples are exposed to the same Space environmental factors, except for microgravity. On the ISS, centrifuges suitable for small samples are available for performing 1-g controls and, more recently, a system for long-term rodent investigations has also been implemented [80,81]. Lack of in-flight 1-g controls leads to difficulties in discerning between the effects of microgravity from other aspects of spaceflight, such as vibration, radiation exposure, etc [82]. Moreover, controls are important to identify the effects due to hardware design or sample conditions, like alterations in gas exchange (due to the lack of convection in Space) and temperature control, among others. For example, early studies in plant response to microgravity were compromised by the build-up of the gaseous hormone ethylene and the lack of appropriate controls [82,83].

\subsubsection{Experimental Design}

Because of the complexity of the ISS environment and the limited flight opportunities, careful experimental design is even more important for spaceflight research. In general, the scientific objectives should be clear, while experiments should be kept as simple as possible. A reduced number of treatments, carefully designed controls, hardware robustness, and sample resilience are all factors that could increase the success of the experiment. Smaller mass and time and energy requirements will contain costs and increase the chances of having a payload approved for flight onboard the ISS. A simple experimental design, accompanied by all the suitable controls, will instead ensure that the conclusions drawn from results are appropriate.

\section{Future Perspective}

Since 1961, when Yuri Gagarin performed the first human flight in Space, multiple short and long duration missions have been conducted on board different Space platforms. In this scenario, it is clear that research in the field of Space biology is crucial for understanding the effects of prolonged permanence in Space on living organisms, and for developing effective countermeasures. Space agencies are taking steps to begin the next era of exploration, to push the boundaries of human exploration forward to the Moon and on to Mars. The first of these steps will be establishing a permanent human presence around the Moon within the next decade with the Lunar Gateway, a Space station in the cis-lunar orbit $[72,84]$.

With the Artemis programme, NASA will enable human missions to the Lunar Gateway and lunar surface from 2024, and will target sustainable lunar exploration by 2028 [84]. The first Artemis mission will be launched in 2021 (uncrewed full system test), followed by Artemis II in 2023 (crewed mission in cis-lunar Space), and will culminate with Artemis III in 2024 with a crewed mission to the lunar surface. Following Artemis III, crews of two crewmembers will fly to the lunar surface annually, and missions will then become of 
four crewmembers in 2028. Gateway crews will perform scientific investigations, human physiology experiments, assess habitation capabilities for future missions, and investigate exploration technologies that require testing in deep Space. When uncrewed, the Gateway will continue to support science and other activities operated from Earth.

The Lunar Gateway and the lunar missions will be the testbed for new tools and equipment that could be used on the 55 million $\mathrm{km}$ trip to Mars, including technologies that could help build self-sustaining outposts away from Earth using in situ resources. Private entities may also utilise the Lunar Gateway through public/private partnerships. This exploration scenario, combined with new private commercial payload delivery services, will also benefit science and academic communities by providing more frequent and lower-cost missions to the Moon and, ultimately, Mars.

Author Contributions: M.D.B., F.F., and C.P. contributed equally to the manuscript. All authors have read and agreed to the published version of the manuscript.

Funding: This research received no external funding.

Data Availability Statement: Data is contained within the article.

Conflicts of Interest: The authors declare no conflict of interest.

\section{References}

1. De la Torre, R.; Sancho, L.G.; Horneck, G.; de los Ríos, A.; Wierzchos, J.; Olsson-Francis, K.; Cockell, C.S.; Rettberg, P.; Berger, T.; de Vera, J.P.P.; et al. Survival of lichens and bacteria exposed to outer space conditions-Results of the Lithopanspermia experiments. Icarus 2010, 208, 735-748. [CrossRef]

2. Jönsson, K.I.; Rabbow, E.; Schill, R.O.; Harms-Ringdahl, M.; Rettberg, P. Tardigrades survive exposure to space in low Earth orbit. Curr. Biol. 2008, 18, 729-731. [CrossRef]

3. De Vera, J.P.; Alawi, M.; Backhaus, T.; Baqué, M.; Billi, D.; Böttger, U.; Berger, T.; Bohmeier, M.; Cockell, C.; Demets, R.; et al. Limits of Life and the Habitability of Mars: The ESA Space Experiment BIOMEX on the ISS. Astrobiology 2019, 19, 145-157. [CrossRef]

4. Horneck, G. Responses of Bacillus subtilis spores to space environment: Results from experiments in space. Orig. Life Evol. Biosph. 1993, 23, 37-52. [CrossRef]

5. Horneck, G.; Klaus, D.M.; Mancinelli, R.L. Space Microbiology. Microbiol. Mol. Biol. Rev. 2010, 74, 121-156. [CrossRef]

6. Onofri, S.; Selbmann, L.; Pacelli, C.; Zucconi, L.; Rabbow, E.; De Vera, J.P. Survival, DNA, and Ultrastructural Integrity of a Cryptoendolithic Antarctic Fungus in Mars and Lunar Rock Analogs Exposed Outside the International Space Station. Astrobiology 2019, 19, 170-182. [CrossRef]

7. Morita, M.T. Directional gravity sensing in gravitropism. Annu. Rev. Plant Biol. 2010, 61, 705-720. [CrossRef] [PubMed]

8. Lang, T.; Van Loon, J.J.W.A.; Bloomfield, S.; Vico, L.; Chopard, A.; Rittweger, J.; Kyparos, A.; Blottner, D.; Vuori, I.; Gerzer, R.; et al. Towards human exploration of space: The THESEUS review series on muscle and bone research priorities. NPJ Microgravity 2017, 3. [CrossRef] [PubMed]

9. Grimm, D.; Grosse, J.; Wehland, M.; Mann, V.; Reseland, J.E.; Sundaresan, A.; Corydon, T.J. The impact of microgravity on bone in humans. Bone 2016, 87, 44-56. [CrossRef]

10. Aubert, A.E.; Larina, I.; Momken, I.; Blanc, S.; White, O.; Prisk, G.K.; Linnarsson, D. Towards human exploration of space: The THESEUS review series on cardiovascular, respiratory, and renal research priorities. NPJ Microgravity 2016, 2, 16031. [CrossRef]

11. Demontis, G.C.; Germani, M.M.; Caiani, E.G.; Barravecchia, I.; Passino, C.; Angeloni, D. Human pathophysiological adaptations to the space environment. Front. Physiol. 2017, 8, 1-17. [CrossRef] [PubMed]

12. Frippiat, J.P.; Crucian, B.E.; de Quervain, D.J.F.; Grimm, D.; Montano, N.; Praun, S.; Roozendaal, B.; Schelling, G.; Thiel, M.; Ullrich, O.; et al. Towards human exploration of space: The THESEUS review series on immunology research priorities. NPJ Microgravity 2016, 2, 16040. [CrossRef] [PubMed]

13. Crucian, B.E.; Choukèr, A.; Simpson, R.J.; Mehta, S.; Marshall, G.; Smith, S.M.; Zwart, S.R.; Heer, M.; Ponomarev, S.; Whitmire, A.; et al. Immune system dysregulation during spaceflight: Potential countermeasures for deep space exploration missions. Front. Immunol. 2018, 9, 1437. [CrossRef]

14. Blaber, A.P.; Zuj, K.A.; Goswami, N. Cerebrovascular autoregulation: Lessons learned from spaceflight research. Eur. J. Appl. Physiol. 2013, 113, 1909-1917. [CrossRef] [PubMed]

15. White, O.; Clément, G.; Fortrat, J.O.; Pavy-Letraon, A.; Thonnard, J.L.; Blanc, S.; Wuyts, F.L.; Paloski, W.H. Towards human exploration of space: The THESEUS review series on neurophysiology research priorities. NPJ Microgravity 2016, $2,16023$. [CrossRef]

16. Roberts, D.R.; Asemani, D.; Nietert, P.J.; Eckert, M.A.; Inglesby, D.C.; Bloomberg, J.J.; George, M.S.; Brown, T.R. Prolonged microgravity affects human brain structure and function. Am. J. Neuroradiol. 2019, 40, 1878-1885. [CrossRef]

17. Bergouignan, A.; Stein, T.P.; Habold, C.; Coxam, V.; O'gorman, D.; Blanc, S. Towards human exploration of space: The THESEUS review series on nutrition and metabolism research priorities. NPJ Microgravity 2016, 2, 16029. [CrossRef] 
18. Mulavara, A.P.; Feiveson, A.H.; Fiedler, J.; Cohen, H.; Peters, B.T.; Miller, C.; Brady, R.; Bloomberg, J.J. Locomotor function after long-duration space flight: Effects and motor learning during recovery. Exp. Brain Res. 2010, 202, 649-659. [CrossRef]

19. Sachs, J. Über orthotrope und plagiotrope Pflanzenteile. Arb. Bot. Inst. Wurzbg. 1882, 2, 226-284.

20. Dedolph, R.R.; Dipert, M.H. The Physical Basis of Gravity Stimulus Nullification by Clinostat Rotation. Plant Physiol. 1971, 47, 756-764. [CrossRef]

21. Cogoli, M. The fast rotating clinostat: A history of its use in gravitational biology and a comparison of ground-based and flight experiment results. ASGSB Bull. 1992, 5, 59-67. [PubMed]

22. Shen-Miller, J.; Hinchman, R.; Gordon, S.A. Thresholds for Georesponse to Acceleration in Gravity-Compensated Avena Seedlings. Plant Physiol. 1968, 43, 338-344. [CrossRef]

23. Galland, P.; Finger, H.; Wallacher, Y. Gravitropism in Phycomyces: Threshold determination on a clinostat centrifuge. J. Plant Physiol. 2004, 161, 733-739. [CrossRef] [PubMed]

24. Laurinavičius, R.; Švegždiene, D.; Buchen, B.; Sievers, A. Determination of the threshold acceleration for the gravitropic stimulation of cress roots and hypocotyls. Adv. Sp. Res. 1998, 21, 1203-1207. [CrossRef]

25. Chauvet, H.; Pouliquen, O.; Forterre, Y.; Legué, V.; Moulia, B. Inclination not force is sensed by plants during shoot gravitropism. Sci. Rep. 2016, 6, 1-8. [CrossRef]

26. Kiss, J.Z.; Wolverton, C.; Wyatt, S.E.; Hasenstein, K.H.; van Loon, J.J.W.A. Comparison of Microgravity Analogs to Spaceflight in Studies of Plant Growth and Development. Front. Plant Sci. 2019, 10, 1577. [CrossRef]

27. van Loon, J.J.W.A. Some history and use of the random positioning machine, RPM, in gravity related research. Adv. Sp. Res. 2007, 39, 1161-1165. [CrossRef]

28. Herranz, R.; Anken, R.; Boonstra, J.; Braun, M.; Christianen, P.C.M.; De Geest, M.; Hauslage, J.; Hilbig, R.; Hill, R.J.A.; Lebert, M.; et al. Ground-based facilities for simulation of microgravity: Organism-specific recommendations for their use, and recommended terminology. Astrobiology 2013, 13, 1-17. [CrossRef]

29. Brungs, S.; Egli, M.; Wuest, S.L.; Peter, P.C.; Jack, J.J.; Ngo Anh, T.J.; Hemmersbach, R. Facilities for Simulation of Microgravity in the ESA Ground-Based Facility Programme. Microgravity Sci. Technol. 2016, 28, 191-203. [CrossRef]

30. Wuest, S.L.; Richard, S.; Kopp, S.; Grimm, D.; Egli, M. Simulated Microgravity: Critical Review on the Use of Random Positioning Machines for Mammalian Cell Culture. Biomed. Res. Int. 2015, 2015, 97147. [CrossRef]

31. Wuest, S.L.; Stern, P.; Casartelli, E.; Egli, M. Fluid dynamics appearing during simulated microgravity using Random Positioning Machines. PLoS ONE 2017, 12, e0170826. [CrossRef] [PubMed]

32. Leguy, C.A.D.; Delfos, R.; Pourquie, M.J.B.M.; Poelma, C.; Krooneman, J.; Westerweel, J.; Van Loon, J.J.W.A. Fluid motion for microgravity simulations in a random positioning machine. Gravit. Sp. Biol. 2011, 25, 36-39.

33. Unsworth, B.R.; Lelkes, P.I. Growing tissues in microgravity. Nat. Med. 1998, 4, 901-907. [CrossRef] [PubMed]

34. Grimm, D.; Wehland, M.; Pietsch, J.; Aleshcheva, G.; Wise, P.; Van Loon, J.; Ulbrich, C.; Magnusson, N.E.; Infanger, M.; Bauer, J. Growing tissues in real and simulated microgravity: New methods for tissue engineering. Tissue Eng. Part B Rev. 2014, 20, 555-566. [CrossRef]

35. Borst, A.G.; Van Loon, J.J.W.A. Technology and developments for the random positioning machine, RPM. Microgravity Sci. Technol. 2009, 21, 287-292. [CrossRef]

36. Herranz, R.; Larkin, O.J.; Dijkstra, C.E.; Hill, R.J.A.; Anthony, P.; Davey, M.R.; Eaves, L.; van Loon, J.J.W.A.; Medina, F.J.; Marco, R. Microgravity simulation by diamagnetic levitation: Effects of a strong gradient magnetic field on the transcriptional profile of Drosophila melanogaster. BMC Genom. 2012, 13, 1-13. [CrossRef]

37. Valles, J.M.; Maris, H.J.; Seidel, G.M.; Tang, J.; Yao, W. Magnetic levitation-based Martian and Lunar gravity simulator. Adv. Sp. Res. 2005, 36, 114-118. [CrossRef]

38. Glover, P.M.; Cavin, I.; Qian, W.; Bowtell, R.; Gowland, P.A. Magnetic-field-induced vertigo: A theoretical and experimental investigation. Bioelectromagnetics 2007, 28, 349-361. [CrossRef]

39. Maret, G.; Dransfeld, K. Biomolecules and Polymers in High Steady Magnetic Fields. In Strong and Ultrastrong Magnetic Fields and Their Applications; Springer: Berlin/Heidelberg, Germany, 1985; pp. 143-204.

40. Nechitailo, G.S.; Mashinsky, A.L.; Kuznetsov, A.A.; Chikov, V.M.; Kuznetsov, O.A. Influence of nonuniform magnetic fields on orientation of plant seedlings in microgravity conditions. Adv. Sp. Res. 2001, 28, 639-643. [CrossRef]

41. Zablotskii, V.; Polyakova, T.; Lunov, O.; Dejneka, A. How a High-Gradient Magnetic Field Could Affect Cell Life. Sci. Rep. 2016, 6, 1-13. [CrossRef]

42. Ploutz-Snyder, L. Evaluating countermeasures in spaceflight analogs. J. Appl. Physiol. 2016, 120, 915-921. [CrossRef] [PubMed]

43. Pavy-Le Traon, A.; Sigaudo, D.; Vasseur, P.; Maillet, A.; Fortrat, J.O.; Hughson, R.L.; Gauquelin-Koch, G.; Gharib, C. Cardiovascular responses to orthostatic tests after a 42-day head-down bed-rest. Eur. J. Appl. Physiol. Occup. Physiol. 1997, 77, 50-59. [CrossRef] [PubMed]

44. Nicogossian, A.E.; Huntoon, C.; Pool, S. Space Physiology and Medicine; Lea and Febiger: Philadelphia, PA, USA, 1994; ISBN 9780812115956.

45. Hoson, T. Apoplast as the site of response to environmental signals. J. Plant Res. 1998, 111, 167-177. [CrossRef]

46. Hoson, T.; Soga, K. New aspects of gravity responses in plant cells. Int. Rev. Cytol. 2003, 229, 209-244. [CrossRef] [PubMed]

47. Navasiolava, N.M.; Custaud, M.A.; Tomilovskaya, E.S.; Larina, I.M.; Mano, T.; Gauquelin-Koch, G.; Gharib, C.; Kozlovskaya, I.B. Long-term dry immersion: Review and prospects. Eur. J. Appl. Physiol. 2011, 111, 1235-1260. [CrossRef] 
48. Tomilovskaya, E.; Shigueva, T.; Sayenko, D.; Rukavishnikov, I.; Kozlovskaya, I. Dry immersion as a ground-based model of microgravity physiological effects. Front. Physiol. 2019, 10, 1-17. [CrossRef] [PubMed]

49. Leach-Huntoon, C.; Grigoriev, A.; Natochin, Y. Fluid and Electrolyte Regulation in Spaceflight; American Astronautical Society: San Diego, CA, USA, 1998; ISBN 978-0877034438.

50. Tesch, P.A.; Lundberg, T.R.; Fernandez-Gonzalo, R. Unilateral lower limb suspension: From subject selection to "omic" responses. J. Appl. Physiol. 2016, 120, 1207-1214. [CrossRef] [PubMed]

51. Globus, R.K.; Morey-Holton, E. Hindlimb unloading: Rodent analog for microgravity. J. Appl. Physiol. 2016, 120, 1196-1206. [CrossRef] [PubMed]

52. Palus, S.; Springer, J.I.; Doehner, W.; von Haehling, S.; Anker, M.; Anker, S.D.; Springer, J. Models of sarcopenia: Short review. Int. J. Cardiol. 2017, 238, 19-21. [CrossRef] [PubMed]

53. Grindeland, R.E.; Ballard, R.W.; Connolly, J.P.; Vasques, M.F. COSMOS 2044 mission. Overview. J. Appl. Physiol. 1992, 73, 1S-3S. [CrossRef]

54. Grindeland, R. Comparison of hypergravity and microgravity effects on rat physiology: An overview. Aviat Sp. Environ. Med. 1998, 69, 59 .

55. Riley, D.A.; Ellis, S.; Giometti, C.S.; Hoh, J.F.Y.; Ilyina-Kakueva, E.I.; Oganov, V.S.; Slocum, G.R.; Bain, J.L.W.; Sedlak, F.R. Muscle sarcomere lesions and thrombosis after spaceflight and suspension unloading. J. Appl. Physiol. 1992, 73, 33S-43S. [CrossRef] [PubMed]

56. Morey-Holton, E.R.; Globus, R.K. Hindlimb unloading rodent model: Technical aspects. J. Appl. Physiol. 2002, 92, 1367-1377. [CrossRef] [PubMed]

57. Qaisar, R.; Karim, A.; Elmoselhi, A.B. Muscle unloading: A comparison between spaceflight and ground-based models. Acta Physiol. 2020, 228, e13431. [CrossRef]

58. 4 Drop Tower-European Space Agency. Available online: http://wsn.spaceflight.esa.int/docs/EUG2LGPr3/EUG2LGPr3-4 -DropTower.pdf (accessed on 23 October 2020).

59. Ruyters, G.; Friedrich, U. From the Bremen Drop Tower to the international space station ISS-Ways to weightlessness in the German Space Life Sciences Program. Signal Transduct. 2006, 6, 397-405. [CrossRef]

60. 5 Parabolic Flights-European Space Agency. Available online: http://wsn.spaceflight.esa.int/docs/EUG2LGPr3/EUG2LGPr3 -5-ParabolicFlights.pdf (accessed on 23 October 2020).

61. Shelhamer, M. Parabolic flight as a spaceflight analog. J. Appl. Physiol. 2016, 120, 1442-1448. [CrossRef]

62. 6 Sounding Rockets-European Space Agency. Available online: http://wsn.spaceflight.esa.int/docs/EUG2LGPr3/EUG2LGPr3 -6-SoundingRockets.pdf (accessed on 23 October 2020).

63. NASA Sounding Rocket Program Overview. Available online: https://rscience.gsfc.nasa.gov/srrov.html (accessed on 29 October 2020).

64. Santoni, F.; Gugliermetti, L.; Piras, G.; De Pascale, S.; Pannico, A.; Piergentili, F.; Marzioli, P.; Frezza, L.; Amadio, D.; Gianfermo, A.; et al. GreenCube: Microgreens cultivation and growth monitoring on-board a 3U CubeSat. In Proceedings of the Conference: 2020 IEEE 7th International Workshop on Metrology for AeroSpace, Pisa, Italy, 22-24 June 2020; pp. 130-135. [CrossRef]

65. Meneghin, A.; Brucato, J.R.; Poggiali, G.; Nascetti, A.; Anfossi, L.; Mirasoli, M. Astrobio Cubesat: A Mini Laboratory Payload for Space Environment Astrobiology Experiments. In Proceedings of the EANA 2019-19th EANA Astrobiology Conference, Orléans, France, 3-6 September 2019; pp. 2-3. [CrossRef]

66. Nicholson, W.L.; Ricco, A.J.; Agasid, E.; Beasley, C.; Diaz-Aguado, M.; Ehrenfreund, P.; Friedericks, C.; Ghassemieh, S.; Henschke, M.; Hines, J.W.; et al. The O/OREOS mission: First science data from the Space Environment Survivability of Living Organisms (SESLO) payload. Astrobiology 2011, 11, 951-958. [CrossRef]

67. Ricco, A.J.; Hanel, R.; Bhattacharya, S.; Boone, T.; Tan, M.; Mousavi, A.; Padgen, M.; Gentry, D.; Rademacher, A.; Schooley, A.; et al. The biosentinel bioanalytical microsystem: Characterizing DNA radiation damage in living organisms beyond earth orbit. In Proceedings of the Solid-State Sensors, Actuators and Microsystems Workshop Hilton Head Island, Hilton Head Island, SC, USA, 5-9 June 2016; pp. 352-355. [CrossRef]

68. Polat, H.C.; Virgili-Llop, J.; Romano, M. Survey, Statistical Analysis and Classification of Launched CubeSat Missions with Emphasis on the Attitude Control Method. JoSS 2016, 5, 513-530.

69. Lemmer, K. Propulsion for CubeSats. Acta Astronaut. 2017, 134, 231-243. [CrossRef]

70. Marinan, A.; Nicholas, A.; Cahoy, K. Ad hoc CubeSat constellations: Secondary launch coverage and distribution. In Proceedings of the IEEE Aerospace Conference Proceedings, Big Sky, MT, USA, 2-9 March 2013. [CrossRef]

71. Schoolcraft, J.; Klesh, A.; Werne, T. MarCO: Interplanetary mission development on a cubesat scale. AIAA 2016, 1-8. [CrossRef]

72. The Global Exploration Roadmap 2018. Available online: https://www.globalspaceexploration.org/wordpress/wp-content/ isecg/GER_2018_small_mobile.pdf (accessed on 23 October 2020).

73. ISS Benefits for Humanity—3rd Edition NASA. Available online: https://www.nasa.gov/mission_pages/station/research/ news/b4h-3rd-ed-book/ (accessed on 23 October 2020).

74. Bryce, C.C.; Horneck, G.; Rabbow, E.; Edwards, H.G.M.; Cockell, C.S. Impact shocked rocks as protective habitats on an anoxic early Earth. Int. J. Astrobiol. 2015, 14, 115-122. [CrossRef]

75. Mancinelli, R.L. The affect of the space environment on the survival of Halorubrum chaoviator and Synechococcus (Nägeli): Data from the Space Experiment OSMO on EXPOSE-R. Int. J. Astrobiol. 2015, 14, 123-128. [CrossRef] 
76. Neuberger, K.; Lux-Endrich, A.; Panitz, C.; Horneck, G. Survival of spores of trichoderma longibrachiatum in space: Data from the Space Experiment SPORES on EXPOSE-R. Int. J. Astrobiol. 2015, 14, 129-135. [CrossRef]

77. Panitz, C.; Horneck, G.; Rabbow, E.; Rettberg, P.; Moeller, R.; Cadet, J.; Douki, T.; Reitz, G. The SPORES experiment of the EXPOSE-R mission: Bacillus subtilis spores in artificial meteorites. Int. J. Astrobiol. 2015, 14, 105-114. [CrossRef]

78. Annual Highlights of Results from the International Space Station (1 October 2018-1 October 2019). Available online: https: //www.nasa.gov/sites/default/files/atoms / files/np-2019-11-010-jsciss_annual_highlights_2019_screen_12-11-19.pdf (accessed on 26 October 2020).

79. Paul, A.; Ferl, R. Spaceflight exploration in plant gravitational biology. Methods Mol. Biol. 2015, 1309, 285-305. [CrossRef]

80. Shiba, D.; Mizuno, H.; Yumoto, A.; Shimomura, M.; Kobayashi, H.; Morita, H.; Shimbo, M.; Hamada, M.; Kudo, T.; Shinohara, M.; et al. Development of new experimental platform 'MARS'-Multiple Artificial-gravity Research System-to elucidate the impacts of micro/partial gravity on mice. Sci. Rep. 2017, 7, 1-10. [CrossRef]

81. Brinckmann, E. Centrifuges and their application for biological experiments in space. Microgravity Sci. Technol. 2012, 24, 365-372. [CrossRef]

82. Vandenbrink, J.P.; Kiss, J.Z. Space, the final frontier: A critical review of recent experiments performed in microgravity. Plant Sci. 2016, 243, 115-119. [CrossRef]

83. Kiss, J.Z.; Edelmann, R.E.; Wood, P.C. Gravitropism of hypocotyls of wild-type and starch-deficient Arabidopsis seedlings in spaceflight studies. Planta 1999, 209, 96-103. [CrossRef]

84. Global Exploration Roadmap Supplement 2020. Available online: https://www.globalspaceexploration.org/wp-content/ uploads/2020/08/GER_2020_supplement.pdf (accessed on 23 October 2020). 